\title{
O COMPORTAMENTO FOTOQUÍMICO DOS COMPLEXOS PENTAAMINAS DE RUTÊNIO(II) COM LIGANTES
} $\pi$ INSATURADOS

Rose Maria Carlos $\$$

Instituto de Química de Araraquara - Universidade Estadual Paulista "Júlio de Mesquita" - Araraquara - SP

Elia Tfouni

Faculdade de Filosofia, Ciências e Letras de Riberão Preto - Universidade de São Paulo - Av. dos Bandeirantes - 3900 - 14040-901

Riberão Preto - SP

Miguel G. Neumann ${ }^{\#}$

Instituto de Química de São Carlos - Universidade de São Paulo - CP 780 - 13560-970 - São Carlos - SP

Recebido em 28/11/95; aceito em 9/1/97

\begin{abstract}
THE PHOTOCHEMICAL BEHAVIOUR OF PENTAAMINERUTHENIUM(II) COMPLEXES WITH

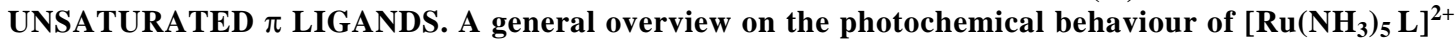
complexes (where $L$ is a $\pi$ ligand) is presented. The proposed mechanisms and techniques employed for the study of these reactions are discussed. Emphasis is made on the mechanisms that allow the identification of the reactive excited state of the $\left[\mathrm{Ru}\left(\mathrm{NH}_{3}\right)_{5} \mathrm{py}\right]^{2+}$ complex.
\end{abstract}

Keywords: photochemistry; photochemical reaction mechanisms; sensitization.

\section{INTRODUÇÃO}

As pesquisas em fotoquímica inorgânica têm apresentado um progresso extraordinário tanto em área de atuação como em importância, após a publicação da monografia ${ }^{1}$ de Balzani e Carassiti em 1970. Nesse período, foram realizados estudos fotoquímicos intensivos ${ }^{1,2}$ dos complexos de aminas de $\mathrm{Co}(\mathrm{III})$, $\mathrm{Pt}(\mathrm{IV})$ e $\mathrm{Cr}(\mathrm{III})$. Esses estudos levaram à proposição de regras empíricas ${ }^{3}$ para prever quais dos seis ligantes ao redor do centro metálico hexacoordenado seriam labilizados na fotólise de campo ligante. Durante a década de 70 , o campo de atuação da fotoquímica dos complexos de metais de transição cresceu rapidamente e em muitas direções. Foram descobertos novos fenômenos, tais como a fotossensibilização dos complexos de aminas de $\mathrm{Co}(\mathrm{III})$ e a emissão dos complexos de $\mathrm{Cr}$ (III) a temperatura ambiente e em solução aquosa ${ }^{4,5}$. Esta última observação fez com que estudos de supressão de luminescência fossem possíveis ${ }^{6}$. Esses estudos levaram a investigações mais detalhadas e sofisticadas dos processos que ocorrem nos estados excitados ${ }^{7}$, tais como as reações de transferência de elétrons e de energia ${ }^{8}$, estimulando as investigações dos processos de conversão de energia solar em energia elétrica ou química ${ }^{9}$.

Também foi estudada a fotoquímica de novas famílias de complexos do tipo Werner ${ }^{10}$, tais como os de $\mathrm{Rh}(\mathrm{III}), \mathrm{Ir}(\mathrm{III})$ e $\mathrm{Ru}(\mathrm{II})$, dos complexos com polipiridinas, e dos sistemas envolvendo $\mathrm{S}$ e $\mathrm{P}$ coordenados com novos tipos de macrociclos, incluindo aqueles de interesse biológico ${ }^{11}$. Por outro lado, foi dada grande atenção a toda a área de compostos organometálicos ${ }^{12}$.

Os últimos 15 anos podem ser caracterizados pelo desenvolvimento e aplicação de novas técnicas (por exemplo, fotólise relâmpago e por pulso de laser nos domínios de nano-, pico- e femtossegundos), melhorias nos métodos de pesquisa mais tradicionais (como aplicações de Raman e EPR) e pela orientação das pesquisas em fotoquímica para a solução de problemas de importância prática (síntese de novos compostos, fotocatálise, utilização de energia solar, etc. $)^{13,14}$.

Um complexo metálico no estado excitado pode ser visualizado como uma nova espécie com uma configuração eletrônica

\footnotetext{
${ }^{\S}$ Endereço atual: R. Santa Cruz, 875 - 13560-680 São Carlos SP

"E-mail: neumann@iqsc.sc.usp.br
}

diferente daquela do estado fundamental, mas com a mesma composição química. Isto permite que possam ser usadas argumentações mecanísticas similares para as reações dos estados excitados e do estado fundamental ${ }^{15}$. A proposição de novos mecanismos tornou-se mais interessante e melhor fundamentada pelo uso de técnicas de excitação e deteção rápidas, que permitem medidas diretas das propriedades espectroscópicas e da cinética dos estados excitados ${ }^{16}$.

Em geral, os estudos mecanísticos de fotorreações têm se concentrado em sistemas nos quais não são observadas reações térmicas que compitam com as fotoquímicas, simplificando substancialmente a caracterização dos produtos das fotorreações ${ }^{13,14 a, 16-18}$. Entre eles, os complexos $d^{6}$ de baixo spin representam provavelmente a configuração eletrônica mais estudada entre os complexos de metais de transição ${ }^{17 d, 18}$. Neste contexto, os complexos de aminas de Ru(II) são de interesse tanto por suas características individuais como pela similaridade de seu comportamento com o de outros complexos $\mathrm{nd}^{6}$. Muitos destes complexos têm sido bem caracterizados e suas reatividades térmicas ${ }^{19,20}$ e fotoquímicas têm sido investigadas ${ }^{10,16,18,21-28}$. Uma característica comum a estes compostos são os altos rendimentos quânticos de formação de produtos resultantes da irradiação nas bandas de $\mathrm{LF}^{21-28}$. Em contrapartida, as reações redox subsequentes à irradiação nas bandas de transferência de carga (CT) dos complexos de aminas de rutênio(II) apresentam rendimentos quânticos dependentes do comprimento de onda de irradiação ${ }^{24-26,28}$.

Este artigo pretende revisar as propriedades fotoquímicas dos complexos pentaaminas de $\mathrm{Ru}(\mathrm{II})$ com ligantes $\pi$ insaturados. Os objetivos desta revisão são: (i) fazer uma coletânea dos resultados obtidos nos estudos fotoquímicos destes complexos; (ii) mostrar as similaridades e descontinuidades dos comportamentos fotoquímicos entre os complexos que compõem esta série; e (iii) mostrar a importância da técnica de fotossensibilização na elucidação dos mecanismos de reações fotoquímicas e na avaliação das energias dos estados excitados dos complexos de coordenação.

Por outro lado, a fotoquímica dos complexos de pentaaminas

Siglas. LF: campo ligante; MLCT: transferência de carga metalpara-ligante; CTTS: transferência de carga para solvente 
de $\mathrm{Ru}(\mathrm{II})$ com ligantes heterocíclicos nitrogenados é um campo promissor para o estudo dos mecanismos de reações fotoquímicas inorgânicas ${ }^{18}$. Estes compostos são parte fundamental dos blocos que formam os complexos binucleares, nos quais são estudadas as reações de transferência intramolecular de elétrons, de bastante interesse em sistemas supramoleculares ${ }^{29}$. Além disso, o conjunto de estados excitados da série pentaaminas de rutênio(II) está relacionado aos complexos de polipiridinas de rutênio(II) usados em estudos de conversão de energia solar $^{14}$.

\section{$\left(\mathrm{NH}_{3}\right)_{5} \mathrm{Ru}^{\mathrm{II}} \mathrm{pz} \mathrm{Cu} \mathrm{Cu}^{\mathrm{II}}$}

Os espectros eletrônicos dos complexos de $\mathrm{Ru}(\mathrm{II})$ com ligantes $\pi$ caracterizam-se por uma intensa banda de absorção de transferência de carga metal-para-ligante (MLCT) ${ }^{10 \mathrm{~b}, 18,19}$. Isto acontece também para as pentaaminas de $\mathrm{Ru}(\mathrm{II})$, $\left[\left(\mathrm{NH}_{3}\right)_{5} \mathrm{RuL}\right]^{2+}$. Quando L é um ligante heterocíclico nitrogenado aromático ${ }^{19 \mathrm{a}}$, uma nitrila tal como benzonitrila ou acetonitrila ${ }^{30}$, ou mesmo dinitrogênio $\left(\mathrm{N}_{2}\right)^{31}$, são observadas bandas de absorção de MLCT intensas nos espectros eletrônicos.

A irradiação de alguns complexos, induz processos fotoquímicos consistentes com a formulação dos estados de MLCT, com o centro metálico oxidado coordenado a um radical $^{32}$ $\left[\left(\mathrm{NH}_{3}\right)_{5} \mathrm{Ru}^{\mathrm{III}}\left(\mathrm{L}^{-}\right)\right]^{2+}$. Como exemplo, pode-se citar a transferência de elétrons estimulada fotoquimicamente, observada no complexo binuclear pentaaminpirazinarutênio(II) $\operatorname{com~} \mathrm{Cu}^{2+}$.

\section{$\left[\mathrm{Ru}\left(\mathrm{NH}_{3}\right)_{5} \mathrm{pz}\right]^{2+}, \quad \mathrm{pz}=\mathrm{N} \mathrm{N}$}

O ligante orgânico apresenta um segundo sítio de coordenação com propriedades ácidas, configurando uma base apropriada para ligar íons hidrogênio ou outros cátions metálicos ${ }^{33}$

$\underset{A}{\left[\left(\mathrm{NH}_{3}\right)_{5} \mathrm{Ru} \mathrm{pz}\right]^{2+}+\mathrm{M}^{\mathrm{n}+} \rightleftharpoons\left[\left(\mathrm{NH}_{3}\right)_{5} \mathrm{RupzM}_{\mathrm{B}}^{(\mathrm{n}+2)+}\right.}$

$\mathrm{M}^{\mathrm{n}+}=\mathrm{H}^{+}, \mathrm{Cu}^{2+}, \mathrm{Zn}^{2+}, \mathrm{Ni}^{2+}$

Sob fotólise contínua, tanto o complexo com pirazina (A) como seu análogo protonado (B) não são reativos em relação à fotossubstituição ${ }^{32}$. No entanto, em experimentos de fotólise relâmpago, o complexo $\mathrm{B}$ com $\mathrm{M}^{\mathrm{n}+}=\mathrm{Cu}^{2+}$ mostra a formação de transientes, seguida da regeneração do composto inicial ${ }^{32}$. A formação desse transiente foi interpretada como resultado da transferência fotoinduzida de elétrons

$\left(\mathrm{NH}_{3}\right)_{5} \mathrm{Ru}^{\mathrm{II}} \mathrm{pz} \mathrm{Cu} \stackrel{\mathrm{h} \nu}{\rightleftharpoons}\left(\mathrm{NH}_{3}\right)_{5} \mathrm{Ru}^{\mathrm{III}} \mathrm{pz} \mathrm{Cu}^{\mathrm{I}}$

A transferência reversa de elétrons regenera o complexo inicial, caracterizando um processo fotocrômico.

Toma e Santos ${ }^{16 c}$ fizeram estudos de ressonância Raman do complexo de $\mathrm{Ru}$ (II) com pirazina para avaliar o mecanismo proposto para a transferência de elétrons observada anteriormente. Enquanto o espectro de absorção do complexo Ru(II)-pz é caracterizado $^{19 a}$ por uma banda intensa de absorção de MLCT $\left(\lambda_{\max }=472 \mathrm{~nm}, \varepsilon=13300 \mathrm{M}^{-1} \mathrm{~cm}^{-1}\right.$ para $\left.\left[\mathrm{Ru}\left(\mathrm{NH}_{3}\right)_{5} \mathrm{pz}\right]^{2+}\right)$, nos espectros de absorção dos complexos Ru(III)-pz estas bandas são bem menos intensas ${ }^{19 b}\left(\lambda=270 \mathrm{~nm}, \varepsilon=0,70 \mathrm{M}^{-1} \mathrm{~cm}^{-1} ; \lambda=\right.$ $230 \mathrm{~nm}, \varepsilon=0,60 \mathrm{M}^{-1} \mathrm{~cm}^{-1}$ para $\left.\left[\mathrm{Ru}\left(\mathrm{NH}_{3}\right)_{5} \mathrm{pz}\right]^{3+}\right)$. Assim, seria esperado um aumento de ressonância das bandas Raman para o ligante pz quando estivesse coordenado a Ru(II) e um decréscimo quando estivesse coordenado a Ru(III). Portanto, um decréscimo nas intensidades das bandas Raman de $\left[\left(\mathrm{NH}_{3}\right)_{5} \mathrm{Ru}^{\mathrm{II}} \mathrm{pzCu}{ }^{\mathrm{II}}\right]$ deveria estar associado ao processo de transferência fotoinduzida de elétrons proposto anteriormente por Ford. Experimentalmete, foi observado o desaparecimento das bandas Raman para $\left[\left(\mathrm{NH}_{3}\right)_{5} \mathrm{Ru}^{\mathrm{II}} \mathrm{pz}\right] \mathrm{Cu}^{\mathrm{II}}$ em sistemas com cela fixa enquanto que nenhuma alteração foi detectada quando o sistema era estudado em cela rotatória. Os autores ${ }^{16 c}$ argumentaram que, sob as condições empregadas com a cela fixa, o processo de transferência fotoinduzida de elétrons (eq 2) poderia tornar-se quase saturado, formando produtos de $\mathrm{Ru}(\mathrm{III})$-pz os quais não exibiriam efeitos de ressonância Raman. Assim, propuseram que as mudanças nas intensidades das bandas Raman devem surgir principalmente de um processo fotocrômico de transferência de elétrons.

Apesar deste exemplo, onde se constatou que os estados excitados de MLCT podem levar a processos redox, os comportamentos fotoquímicos mais comuns, geralmente observados nos complexos de aminas de $\mathrm{Ru}(\mathrm{II})$ com ligantes $\pi$ insaturados, são a fotossubstituição dos ligantes e/ou a fotooxidação de $\mathrm{Ru}(\mathrm{II})$ a $\mathrm{Ru}(\mathrm{III})$ com formação simultânea de $\mathrm{H}_{2}$. Estas reações são atribuídas à população de estados excitados de campo ligante $(\mathrm{LF})^{21-28}$ e a estados excitados de transferência de carga-para-solvente (CTTS) $)^{25,26,28}$, respectivamente.

\section{$\left[\operatorname{Ru}\left(\mathrm{NH}_{3}\right)_{5} \mathbf{N}_{2}\right]^{2+}$}

No primeiro estudo fotoquímico de uma amina de $\mathrm{Ru}(\mathrm{II})$ realizado por Sigwart e Spence ${ }^{34}$, foi observado que a irradiação do complexo $\left[\mathrm{Ru}\left(\mathrm{NH}_{3}\right)_{5} \mathrm{~N}_{2}\right]^{2+}\left(\lambda_{\max }=221 \mathrm{~nm}, \varepsilon=16500\right.$ $\mathrm{M}^{-1} \mathrm{~cm}^{-1}$ ) com luz branca levava à oxidação do centro metálico

$\left[\mathrm{Ru}^{\mathrm{II}}\left(\mathrm{NH}_{3}\right)_{5} \mathrm{~N}_{2}\right]^{2+}+\mathrm{X}^{-} \stackrel{\mathrm{hv}}{\longrightarrow}\left[\mathrm{Ru}^{\mathrm{III}}\left(\mathrm{NH}_{3}\right)_{5} \mathrm{X}\right]^{2+}+$ produtos de redução

onde $\mathrm{X}^{-}=\mathrm{Cl}^{-}$em $\mathrm{HCl}$ diluído, ou $\mathrm{OH}^{-}$em solução aquosa neutra. Os produtos de redução podem ser $\mathrm{H}_{2}$ vindo da redução da água, ou formas reduzidas do ligante dinitrogênio.

Estudos fotoquímicos quantitativos ${ }^{25}$ posteriores deste complexo confirmaram a oxidação de $\mathrm{Ru}(\mathrm{II})$ para $\mathrm{Ru}(\mathrm{III})$. A fotólise a $254 \mathrm{~nm}$ de $\left[\mathrm{Ru}\left(\mathrm{NH}_{3}\right)_{5} \mathrm{~N}_{2}\right]^{2+}$ levou a um decréscimo contínuo da banda de MLCT com um rendimento quântico de consumo de $\left[\mathrm{Ru}\left(\mathrm{NH}_{3}\right)_{5} \mathrm{~N}_{2}\right]^{2+}$ de $0,18 \mathrm{~mol} /$ einstein, medido a $240 \mathrm{~nm}$. O complexo $\left[\mathrm{Ru}\left(\mathrm{NH}_{3}\right)_{5} \mathrm{Cl}\right]^{2+}$ também foi observado durante a fotólise mas com rendimento quântico inicial muito baixo $(\Phi<$ $0,01 \mathrm{~mol} /$ einstein). $\mathrm{Na}$ irradiação de $\left[\mathrm{Ru}\left(\mathrm{NH}_{3}\right)_{5} \mathrm{~N}_{2}\right]^{2+}$ a $254 \mathrm{~nm}$ na presença de álcool isopropílico, observou-se a formação de $\mathrm{H}_{2}$ com rendimento quântico ${ }^{25}$ de $0,10 \mathrm{~mol} /$ einstein. Nestas condições, não foi observada a formação de $\left[\mathrm{Ru}\left(\mathrm{NH}_{3}\right)_{5} \mathrm{Cl}\right]^{2+}$ nem mesmo no início da fotólise. Estes resultados sugerem o seguinte mecanismo:

$$
\begin{aligned}
& {\left[\mathrm{Ru}\left(\mathrm{NH}_{3}\right)_{5} \mathrm{~N}_{2}\right]^{2+} \underset{\mathrm{H}_{2} \mathrm{O}}{\longrightarrow}\left[\mathrm{Ru}\left(\mathrm{NH}_{3}\right)_{5} \mathrm{~N}_{2}\right]^{3+}+\mathrm{H}_{2}} \\
& {\left[\mathrm{Ru}\left(\mathrm{NH}_{3}\right)_{5} \mathrm{~N}_{2}\right]^{3+}+\mathrm{H}_{2} \mathrm{O} \longrightarrow\left[\mathrm{Ru}\left(\mathrm{NH}_{3}\right)_{5}\left(\mathrm{H}_{2} \mathrm{O}\right)\right]^{3+}+\mathrm{N}_{2}}
\end{aligned}
$$

Este mecanismo está de acordo com os resultados de Baxendale e Mulazzani ${ }^{35}$, que observaram que nem mesmo a reação de elétrons solvatados com o complexo $\left[\mathrm{Ru}\left(\mathrm{NH}_{3}\right)_{5} \mathrm{~N}_{2}\right]^{2+}$ leva à redução do dinitrogênio.

\section{$\left[\mathrm{Ru}\left(\mathrm{NH}_{3}\right)_{5}\left(\mathrm{CH}_{3} \mathrm{CN}\right)\right]^{2+}$}

Enquanto a fotoquímica do complexo $\left[\mathrm{Ru}\left(\mathrm{NH}_{3}\right)_{5} \mathrm{~N}_{2}\right]^{2+}$ está caracterizada pela oxidação de $\mathrm{Ru}(\mathrm{II})$ a $\mathrm{Ru}(\mathrm{III})$, para $\left[\mathrm{Ru}\left(\mathrm{NH}_{3}\right)_{5}\left(\mathrm{CH}_{3} \mathrm{CN}\right)\right]^{2+}$ é observada uma dependência do rendimento quântico desta reação com o comprimento de onda de irradiação, $\lambda_{\text {irr }}$, sugerindo a existência de dois processos fotoquímicos independentes ${ }^{25}$ : aquação (eq 6 e 7) e oxidação (eq 8):

$$
\left[\mathrm{Ru}\left(\mathrm{NH}_{3}\right)_{5}\left(\mathrm{CH}_{3} \mathrm{CN}\right)\right]^{2+} \stackrel{\text { hv }}{\longrightarrow}\left\{\begin{array}{l}
{\left[\mathrm{Ru}\left(\mathrm{NH}_{3}\right)_{5}\left(\mathrm{H}_{2} \mathrm{O}\right)\right]^{2+}+\mathrm{CH}_{3} \mathrm{CN}} \\
{\left[\mathrm{Ru}\left(\mathrm{NH}_{3}\right)_{4}\left(\mathrm{CH}_{3} \mathrm{CN}\right)\left(\mathrm{H}_{2} \mathrm{O}\right)\right]^{2+}+\mathrm{NH}_{3}} \\
\text { produtos de } \mathrm{Ru}(\mathrm{III})
\end{array}\right.
$$


$\mathrm{O}$ espectro de absorção do complexo $\left[\mathrm{Ru}\left(\mathrm{NH}_{3}\right)_{5}\left(\mathrm{CH}_{3} \mathrm{CN}\right)\right]^{2+}$ apresenta uma banda intensa de $\operatorname{MLCT}^{25,30},\left(\lambda_{\max }=226 \mathrm{~nm}, \varepsilon=\right.$ $\left.15000 \mathrm{M}^{-1} \mathrm{~cm}^{-1}\right)$, análoga à do complexo com dinitrogênio, e uma banda menos intensa a $366 \mathrm{~nm}\left(\varepsilon=150 \mathrm{M}^{-1} \mathrm{~cm}^{-1}\right)$, atribuída a uma transição de campo ligante (LF).

A fotólise de $\left[\mathrm{Ru}\left(\mathrm{NH}_{3}\right)_{5}\left(\mathrm{CH}_{3} \mathrm{CN}\right)\right]^{2+}$ a $\lambda>254 \mathrm{~nm}$, que engloba a banda de absorção de LF, leva predominantemente a reações de substituição dos ligantes coordenados ${ }^{25}$. Os rendimentos quânticos de aquação, $\Phi_{\mathrm{aq}}(\sim 0,30 \mathrm{~mol} /$ einstein $)$ e de oxidação, $\Phi_{\text {ox }}\left(<10^{-3} \mathrm{~mol} /\right.$ einstein $)$ são relativamente independentes do $\lambda_{\text {irr }}$, sugerindo um estado excitado comum, provavelmente de LF. A fotooxidação de $\mathrm{Ru}(\mathrm{II})$ a $\mathrm{Ru}(\mathrm{III})$ foi observada para irradiações na banda de absorção de transferência de carga. Nesta região, os rendimentos quânticos de oxidação aumentam com a energia de irradiação, enquanto que o rendimento quântico de aquação diminui $\left(\Phi_{\mathrm{ox}} \approx 0,40\right.$ e $\Phi_{\mathrm{aq}}<0,03$ mol/einstein, a $230 \mathrm{~nm}$ ). Os espectros deste complexo em vários solventes ${ }^{25}$ mostram a presença de duas bandas: uma na região de $235 \mathrm{~nm}$ e outra a $\sim 270 \mathrm{~nm}$. O deslocamento do máximo em $235 \mathrm{~nm}$ em vários solventes pode ser correlacionado com o esperado para uma banda de MLCT $^{19}$.

Já o máximo a $\sim 270 \mathrm{~nm}$, também depende do meio e tendo um coeficiente de absorção significativo $\left(\varepsilon_{270} \sim 2000 \mathrm{M}^{-1} \mathrm{~cm}^{-1}\right)$, deve corresponder a uma transição diferente ${ }^{25}$. Para $\left[\mathrm{Ru}\left(\mathrm{NH}_{3}\right)_{5}\right.$ $\left.\left(\mathrm{CH}_{3} \mathrm{CN}\right)\right]^{2+}$ esta banda ocorre no mesmo comprimento de onda que para $\left[\mathrm{Ru}\left(\mathrm{NH}_{3}\right)_{6}\right]^{2+}$, apresentando a mesma dependência com o solvente ${ }^{36}$. Estas observações levaram a propor que a banda a $\sim 270 \mathrm{~nm}$ correspondesse a uma transição de CTTS.

O resultado das irradiações a 228 e $214 \mathrm{~nm}$ em presença do álcool isopropílico também é consistente com um estado excitado reativo de $\mathrm{CTTS}^{25}$. O produto predominante destas fotorreações é $\left[\mathrm{Ru}\left(\mathrm{NH}_{3}\right)_{5}\left(\mathrm{CH}_{3} \mathrm{CN}\right)\right]^{3+}$ e o processo pode ser representado por

$$
\begin{aligned}
& {\left[\mathrm{Ru}\left(\mathrm{NH}_{3}\right)_{5}\left(\mathrm{CH}_{3} \mathrm{CN}\right)\right]^{2+} \stackrel{\mathrm{hv}}{\longrightarrow}\left[\mathrm{Ru}\left(\mathrm{NH}_{3}\right)_{5}\left(\mathrm{CH}_{3} \mathrm{CN}\right)\right]^{3+}+\mathrm{e}_{\mathrm{aq}}^{-}} \\
& \mathrm{H}^{+}{ }_{\mathrm{aq}}+\mathrm{e}_{\mathrm{aq}}^{-} \longrightarrow \mathrm{H}_{\mathrm{aq}}^{\cdot} \\
& 2 \mathrm{H}_{\mathrm{aq}}^{\cdot} \longrightarrow \mathrm{H}_{2}
\end{aligned}
$$

A presença do álcool leva a um aumento significativo na formação de $\mathrm{H}_{2}$ e um decréscimo substancial no rendimento quântico de oxidação, de acordo com as eq 12 e 13 .

$$
\begin{aligned}
& \mathrm{H}^{\bullet}+\mathrm{CH}_{3} \mathrm{CH}(\mathrm{OH}) \mathrm{CH}_{3} \longrightarrow \mathrm{H}_{2}+\mathrm{CH}_{3} \dot{\mathrm{C}}(\mathrm{OH}) \mathrm{CH}_{3} \\
& {\left[\mathrm{Ru}\left(\mathrm{NH}_{3}\right)_{5} \mathrm{CH}_{3} \mathrm{CN}\right]^{3+}+\mathrm{CH}_{3} \dot{\mathrm{C}}(\mathrm{OH}) \mathrm{CH}_{3} \longrightarrow} \\
& {\left[\mathrm{Ru}\left(\mathrm{NH}_{3}\right)_{5} \mathrm{CH}_{3} \mathrm{CN}\right]^{2+}+\mathrm{CH}_{3} \mathrm{COCH}_{3}+\mathrm{H}^{+}}
\end{aligned}
$$

A figura 1 mostra os níveis de energia propostos para os estados excitados do complexo $\left[\mathrm{Ru}\left(\mathrm{NH}_{3}\right)_{5}\left(\mathrm{CH}_{3} \mathrm{CN}\right)\right]^{2+}$.

Os rendimentos quânticos para a aquação e a oxidação do complexo foram similares para as irradiações efetuadas a 254 $\mathrm{nm},\left(\Phi_{\mathrm{aq}}=0,12\right.$ e $\Phi_{\mathrm{ox}}=0,16 \mathrm{~mol} /$ einstein $)$. Estes resultados foram interpretados supondo-se a população dos estados excitados de CTTS e de LF.

Os estados excitados $L_{1}$ e MLCT mostrados no diagrama de energia na figura 1 correspondem às bandas observadas no espectro de absorção em solução aquosa. Os estados CTTS e $\mathrm{LF}_{2}$ são representados por linhas pontilhadas uma vez que suas existências foram inferidas ${ }^{25}$. A irradiação na banda de LF leva a um decaimento para o estado de LF de menor energia, a partir do qual ocorre aquação ou desativação não-radiativa para o estado fundamental. Os estados excitados de transferência de carga decaem para o estado de CTTS de menor energia, que leva a oxidação do metal ou desativação não-radiativa para o estado fundamental.

$\mathrm{O}$ comportamento fotoquímico do complexo $\left[\mathrm{Ru}\left(\mathrm{NH}_{3}\right)_{5}\right.$ $\left.\left(\mathrm{CH}_{3} \mathrm{CN}\right)\right]^{2+}$ irradiado a $254 \mathrm{~nm}$ difere do observado para $\left[\mathrm{Ru}\left(\mathrm{NH}_{3}\right)_{5} \mathrm{~N}_{2}\right]^{2+}$. Para os dois complexos observou-se oxidação de $\mathrm{Ru}(\mathrm{II})$ para $\mathrm{Ru}(\mathrm{III})$ com formação simultânea de $\mathrm{H}_{2}$. No

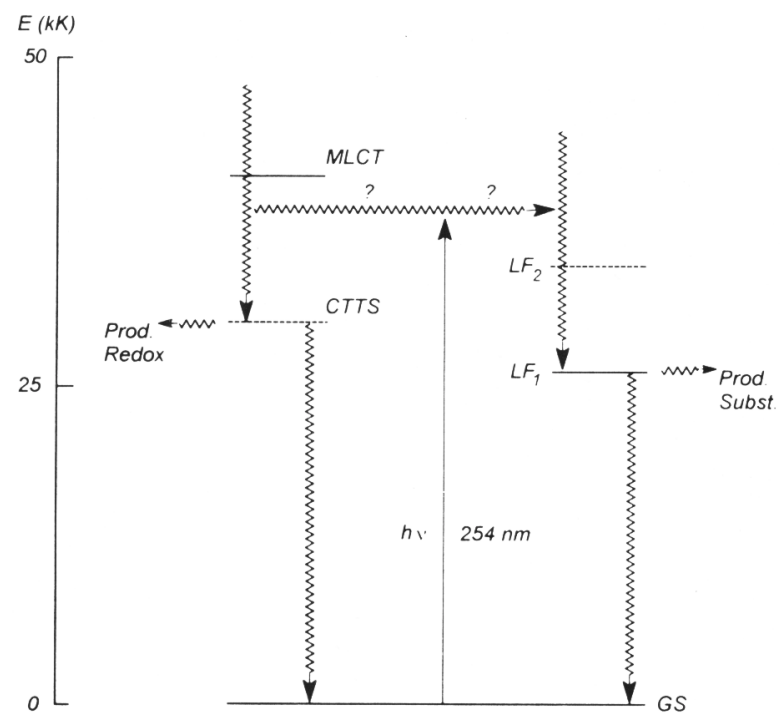

Figura 1. Diagrama de níveis de energia para o complexo $\left[\mathrm{Ru}\left(\mathrm{NH}_{3}\right)_{5}\left(\mathrm{CH}_{3} \mathrm{CN}\right)\right]^{2+}$. MLCT = estado de transferência de carga metal-para-ligante; CTTS = estado de transferência de carga metal-parasolvente $; \mathrm{LF}=$ estado de campo ligante $;$ prod redox $=$ produtos de fotorredução $;$ prod subst $=$ produtos de aquação. $($ Ref. 25$)$.

entanto, enquanto a aquação do ligante $\mathrm{N}_{2}$ é uma consequência da fotooxidação do complexo $\left[\mathrm{Ru}\left(\mathrm{NH}_{3}\right)_{5} \mathrm{~N}_{2}\right]^{2+}$, para $\left[\mathrm{Ru}\left(\mathrm{NH}_{3}\right)_{5}\right.$ $\left.\left(\mathrm{CH}_{3} \mathrm{CN}\right)\right]^{2+}$ a aquação dos ligantes $\mathrm{CH}_{3} \mathrm{CN}$ e $\mathrm{NH}_{3}$ é conseqüência da fotoaquação do complexo.

A fotooxidação de $\mathrm{Ru}(\mathrm{II})$ a $\mathrm{Ru}(\mathrm{III})$ também foi observada nos complexos $\left[\mathrm{Ru}\left(\mathrm{NH}_{3}\right)_{6}\right]^{2+}$ e $\left[\mathrm{Ru}\left(\mathrm{NH}_{3}\right)_{5}\left(\mathrm{H}_{2} \mathrm{O}\right)\right]^{2+}$, apesar destes não apresentarem bandas de MLCT em seus espectros eletrônicos ${ }^{26,36}$. O espectro de $\left[\mathrm{Ru}\left(\mathrm{NH}_{3}\right)_{6}\right]^{2+}$ apresenta uma banda a $275 \mathrm{~nm}\left(\varepsilon=640 \mathrm{M}^{-1} \mathrm{~cm}^{-1}\right)$ e outra mais fraca a $390 \mathrm{~nm}(\varepsilon=$ $\left.35 \mathrm{M}^{-1} \mathrm{~cm}^{-1}\right)$, atribuída ${ }^{26}$ à transição de $\mathrm{LF}^{1} \mathrm{~T}_{\mathrm{gg}} \leftarrow{ }^{1} \mathrm{~A}_{1 \mathrm{~g}}$. O alto valor de $\varepsilon$ da primeira banda sugere características de transferência de carga ${ }^{26}$. Como só ligantes saturados estão coordenados ao centro metálico e, mesmo assim, este complexo apresentou fotooxidação, concluiu-se que esta banda corresponde a uma transição de CTTS $^{26}$. Estudos posteriores de efeito do meio sobre o espectro de absorção do íon $\left[\mathrm{Ru}\left(\mathrm{NH}_{3}\right)_{6}\right]^{2+}$ confirmaram esta atribuição ${ }^{36}$.

A irradiação do complexo $\left[\mathrm{Ru}\left(\mathrm{NH}_{3}\right)_{6}\right]^{2+}$ na região uv-vis em solução aquosa ${ }^{26}$, leva à aquação da amônia e à oxidação de $\mathrm{Ru}(\mathrm{II})$ a $\mathrm{Ru}(\mathrm{III})$ com formação simultânea de $\mathrm{H}_{2}$. A formação destes produtos foi atribuída originalmente ${ }^{26}$ à população de dois estados excitados diferentes, um de LF e outro de CTTS. Para irradiações na região de 313-405 nm, correspondente à banda de absorção de LF, o tipo de reação predominante é a fotoaquação, apesar de também ter sido observada uma pequena proporção de oxidação. Os rendimentos quânticos obtidos são independentes do $\lambda_{\text {irr }}\left(\Phi_{\text {aq }} \sim 0,27\right.$ e $\Phi_{\text {ox }}=0,03 \mathrm{~mol} /$ einstein $^{26}$.

A figura 2 ilustra o modelo de superfícies de energia potencial proposto para esse complexo. A independência dos rendimentos quânticos de aquação e oxidação com o $\lambda_{\text {irr }}$ para irradiações acima de $313 \mathrm{~nm}$, sugere um estado excitado comum a partir do qual se obtêm as reações de aquação e oxidação. Este estado deve ser o estado excitado singlete ${ }^{1} \mathrm{LF}$, uma vez que a região da excitação corresponde à banda de campo ligante. A partir deste estado, o sistema decai para o estado tri-plete de menor energia ${ }^{3} \mathrm{LF}$ por cruzamento entre sistemas. Este estado excitado, que na realidade é um estado com contribuições de triplete e singlete, tem também um certo carácter de CTTS, permitindo uma proporção de oxidação para $\mathrm{Ru}(\mathrm{III})^{28}$. Para irradiações com comprimentos de onda inferiores a $313 \mathrm{~nm}$, correspondente à absorção direta na banda de CTTS, observa-se um aumento significativo do rendimento quântico de oxidação 


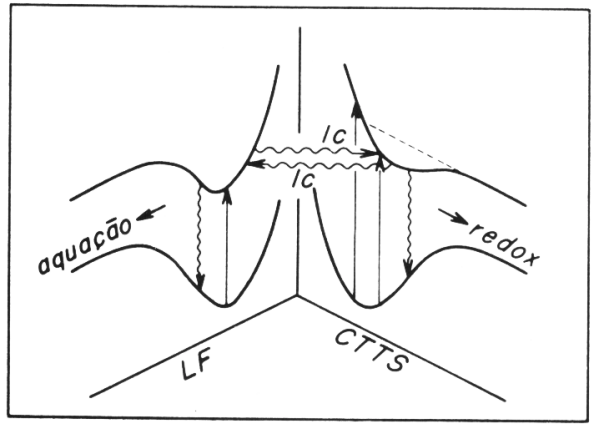

Figura 2. Superfícies de energia potencial para os estados excitados de $\left[\mathrm{Ru}\left(\mathrm{NH}_{3}\right)_{6}\right]^{2+}$, indicando que os processos de aquação e oxidação ocorrem dos estados excitados de campo ligante (LF) e de transferência de carga para solvente (CTTS) e que a desativação não radiativa ocorre independente do estado excitado populado. (Ref. 26).

$\left(\Phi_{\mathrm{ox}}=0,16 \mathrm{~mol} /\right.$ einstein $)$ e um decréscimo no rendimento quântico de aquação ( $\Phi_{\text {aq }}=0,03 \mathrm{~mol} /$ einstein). A observação da aquação nesta região ${ }^{26}$ está de acordo com a proposta acima, só que neste caso o estado CTTS inclui uma certa proporção de caráter de campo ligante.

É interessante notar a diferença entre os comportamentos fotoquímicos de $\left[\mathrm{Ru}\left(\mathrm{NH}_{3}\right)_{6}\right]^{2+}$ e $\left[\mathrm{Ru}\left(\mathrm{NH}_{3}\right)_{5}\left(\mathrm{CH}_{3} \mathrm{CN}\right)\right]^{2+}$. Enquanto que no primeiro ocorrem as reações de aquação e oxidação para irradiações em toda a região do espectro, para o último prevalece a aquação para $\lambda_{\text {irr }}>254 \mathrm{~nm}$ e a oxidação para $\lambda_{\text {irr }}<254 \mathrm{~nm}$.

\section{$\left[\mathrm{Ru}\left(\mathrm{NH}_{3}\right)_{5}(\mathrm{py}-\mathrm{x})\right]^{2+}$}

Nas fotólises dos complexos pentaaminas de Ru(II) com piridina (py) e piridinas substituídas $(p y-x)^{16,21,22,27,28,32}$, $\left[\mathrm{Ru}\left(\mathrm{NH}_{3}\right)_{5}(\mathrm{py}-\mathrm{x})\right]^{2+}$ é obtido um comportamento fotoquímico distinto dos dois observados anteriormente. Os espectros eletrônicos destes complexos são caracterizados por uma banda intensa de $\mathrm{MLCT}^{19}$ na região visível, que depende tanto do solvente como do substituinte $\mathrm{x}$ ligado ao anel da piridina (Tabelas 1 e 2$)^{22}$. Esta dependência em relação ao substituinte pode ser entendida em termos da habilidade do ligante py-x de atuar como aceptor $\pi$. Os substituintes doadores de elétrons aumentam a energia da transição, enquanto que os substituintes aceptores de elétrons diminuem a energia da transição ${ }^{19}$, sendo esta a ordem esperada para uma transição de transferência de carga metal-para-ligante ${ }^{19}$. Em relação à dependência das bandas de absorção com o solvente ${ }^{19 \mathrm{~d}-\mathrm{g}, 22}$, foi observado que a energia da banda de transição eletrônica no visível diminui em solventes doadores de elétrons, indicando interações entre a amônia coordenada e o solvente ${ }^{19 d}$. Estes resultados são consistentes com a atribuição da banda intensa no visível como sendo de MLCT.

A irradiação de $\left[\mathrm{Ru}\left(\mathrm{NH}_{3}\right)_{5}(\mathrm{py}-\mathrm{x})\right]^{2+}$ com luz visível leva a processos de substituição característicos da fotoquímica de campo ligante 21,22 .

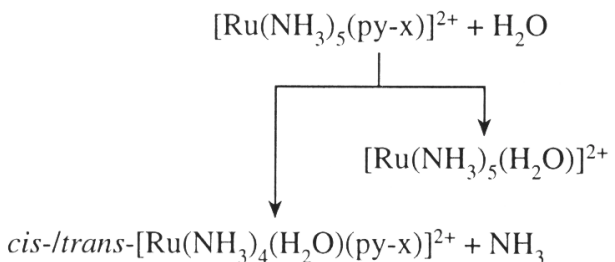

O complexo mais representativo desta série de compostos é $\left[\mathrm{Ru}\left(\mathrm{NH}_{3}\right)_{5} \mathrm{py}\right]^{2+}$, sendo o que mais tem sido estudado ${ }^{10 \mathrm{~b}, 16}$, $21,22,27,32$. O espectro uv-vis de $\left[\mathrm{Ru}\left(\mathrm{NH}_{3}\right)_{5} \mathrm{py}\right]^{2+}$ apresenta uma banda de MLCT a $408 \mathrm{~nm} \quad\left(\varepsilon=7800 \mathrm{M}^{-1} \mathrm{~cm}^{-1}\right)$ e uma banda do ligante a $246 \mathrm{~nm}\left(\varepsilon=4600 \mathrm{M}^{-1} \mathrm{~cm}^{-1}\right)^{19}$. A irradiação de $\left[\mathrm{Ru}\left(\mathrm{NH}_{3}\right)_{5} \mathrm{py}\right]^{2+}$ com luz visível resulta exclusivamente na

Tabela 1. Dados espectroscópicos ${ }^{(\mathrm{a})}$ e rendimentos quânticos de aquação ${ }^{(\mathrm{b})}$ para $\left[\mathrm{Ru}\left(\mathrm{NH}_{3}\right)_{5}(\mathrm{py}-\mathrm{x})\right]^{2+}$.

\begin{tabular}{|c|c|c|c|c|c|}
\hline $\mathrm{X}$ & $\begin{array}{c}\text { banda } * \pi \leftarrow \pi \\
\lambda_{\max }, \mathrm{nm}\left(\varepsilon, \mathrm{M}^{-1} \mathrm{~cm}^{-1}\right)\end{array}$ & $\begin{array}{c}\text { banda MLCT } \\
\lambda_{\max }, \mathrm{nm}\left(\varepsilon, \mathrm{M}^{-1} \mathrm{~cm}^{-1}\right)\end{array}$ & $\lambda_{\text {irr }}, \mathrm{nm}$ & $\Phi_{\mathrm{py}-\mathrm{X}}$ & $\Phi_{\mathrm{NH} 3}$ \\
\hline$p-\mathrm{CH}_{3}$ & $244(4600)$ & 398 (7700) & 405 & 0,037 & \\
\hline$-\mathrm{H}$ & $244(4560)$ & 407 (7780) & 405 & 0,045 & 0,063 \\
\hline$m-\mathrm{Cl}$ & $256(5300)$ & 426 (7900) & 436 & 0,048 & \\
\hline$o-\mathrm{CONH}_{2}$ & $254(5248)$ & $427(6025)$ & 433 & 0,0085 & \\
\hline$-\mathrm{C}_{6} \mathrm{H}_{5}$ & 257 (16982) & 446 (11749) & 449 & 0,033 & 0,019 \\
\hline 3,5-diCl & $267(5495)$ & 447 (9332) & 449 & 0,042 & 0,019 \\
\hline$p-\mathrm{CF}_{3}$ & 254 (4677) & 454 (7244) & 455 & 0,022 & \\
\hline$p-\mathrm{CO}_{2-}$ & 257 (5248) & 457 (6025) & 460 & 0,026 & \\
\hline $\mathrm{pz}$ & 253 (6025) & $472(10715)$ & 479 & 0,0014 & 0,0018 \\
\hline $\mathrm{p}-\mathrm{CONH}_{2}$ & $258(4570)$ & 479 (11470) & 479 & 0,00107 & 0,0053 \\
\hline$p-\mathrm{CO}_{2} \mathrm{CH}_{3}$ & $265(3800)$ & 497 (12598) & 500 & 0,00027 & \\
\hline$p-\mathrm{COCH}_{3}$ & 271 (3388) & $523(9332)$ & 520 & 0,00025 & 0,0009 \\
\hline $\mathrm{pzH}^{+}$ & $272(6456)$ & $529(12022)$ & 520 & 0,00011 & \\
\hline $\mathrm{pzCH}_{3}{ }^{+}$ & $269(6025)$ & $538(12882)$ & 540 & 0,00004 & $<0,0008$ \\
\hline$p$-CHO & 274 (3380) & $545(10332)$ & 546 & 0,00005 & 0,00005 \\
\hline
\end{tabular}

(a) Ford, P. C.; De Rudd, F .P.; Gaunder, R.; Taube, H.; J. Am. Chem. Soc. 1968,90,1187. Malouf, G.; Ford, P. C.; J. Am. Chem. Soc. 1977, 99, 7213.

(b) $\mathrm{A} 25^{\circ} \mathrm{C}$. Em solução $0,2 \mathrm{M}$ de $\mathrm{NaCl}$ a pH $3,0\left(\mathrm{HCl} 10^{-3} \mathrm{M}\right)$ 
Tabela 2. Máximos de absorção da banda de MLCT, $\lambda_{\max }(\mathrm{MLCT})$, e rendimentos quânticos de aquação de $\left[\mathrm{Ru}\left(\mathrm{NH}_{3}\right)_{5}(\mathrm{py}-\mathrm{x})\right]^{2+} \mathrm{em}$ vários solventes para irradiações no máximo de absorção dos complexos. ${ }^{\text {(a) }}$

\begin{tabular}{|c|c|c|}
\hline $\mathrm{X}$ & $\lambda \max (\mathrm{MLCT}), \mathrm{nm}$ & $\Phi, \mathrm{mol} / \mathrm{einstein}$ \\
\hline \multicolumn{3}{|c|}{ Dimetilsulfóxido } \\
\hline$-\mathrm{H}$ & 447 & 0,048 \\
\hline 3,5-diCl & 491 & 0,078 \\
\hline$-\mathrm{C}_{6} \mathrm{H}_{5}$ & 497 & 0,016 \\
\hline$p-\mathrm{CONH}_{2}$ & 511 & 0,0105 \\
\hline$p-\mathrm{COCH}_{3}$ & 566 & 0,0098 \\
\hline \multicolumn{3}{|c|}{ Dimetilformamida } \\
\hline$-\mathrm{H}$ & 436 & 0,036 \\
\hline 3,5-diCl & 482 & 0,0051 \\
\hline$-\mathrm{C}_{6} \mathrm{H}_{5}$ & 487 & 0,0058 \\
\hline$p-\mathrm{CONH}_{2}$ & 501 & 0,0039 \\
\hline \multicolumn{3}{|c|}{ Acetonitrila } \\
\hline$-\mathrm{H}$ & 407 & 0,140 \\
\hline 3,5-diCl & 449 & 0,122 \\
\hline$p-\mathrm{CONH}_{2}$ & 468 & 0,062 \\
\hline$p-\mathrm{COCH}_{3}$ & 504 & 0,036 \\
\hline$p-\mathrm{CHO}$ & 523 & 0,0092 \\
\hline
\end{tabular}

(a) A $25^{\circ}$ C. Malouf, G., Ford, P. C.; J. Am. Chem. Soc. 1977, $99,7213$.

aquação da amônia e da piridina coordenadas $\left(\Phi_{\mathrm{NH} 3}=0,063\right.$ e $\Phi_{\text {py }}=0,045 \mathrm{~mol} /$ einstein $^{21}$, independente do comprimento de onda usado. Por outro lado, o $\Phi_{\text {py }}$ depende fortemente do $\mathrm{pH}$ da solução, enquanto que o $\Phi_{\mathrm{NH} 3}$ é independente do $\mathrm{pH}$, como é mostrado na figura $3^{21 \mathrm{c}}$.

A sensibilidade ao $\mathrm{pH}$ da aquação da piridina não é devida à protonação no estado fundamental, uma vez que o espectro de absorção antes da fotólise independe da concentração do ácido $^{21 \mathrm{c}}$. Também não é devida à reação térmica de volta ${ }^{21 \mathrm{c}}$, uma vez que este efeito deveria ser mais pronunciado a $\mathrm{pH}$ próximo ao $\mathrm{pKa}(5,3)$ da piridina ${ }^{37}$. Estes resultados sugerem caminhos de reação competitivos: um catalisado por ácido e o outro independente do ácido ${ }^{21 c, 22}$.

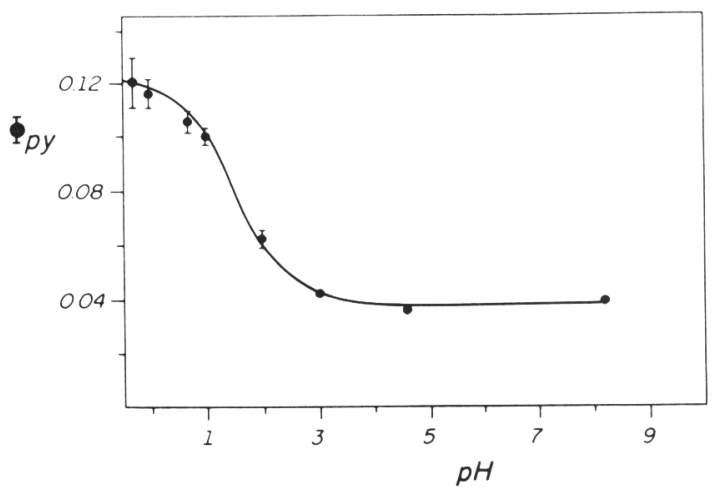

Figura 3. Dependência do rendimento quântico com o pH para a fotoaquação da piridina do $\left[\mathrm{Ru}\left(\mathrm{NH}_{3}\right)_{5} \mathrm{py}\right]^{2+}$ irradiado a $405 \mathrm{~nm} \mathrm{em}$ solução aquosa $25^{\circ} \mathrm{C}$, na presença de íon cloreto. (Ref. 21c).
A irradiação na região da banda de transferência de carga Ru-py envolve a população inicial de um estado excitado de MLCT. Este estado excitado corresponde a um centro metálico oxidado, $\mathrm{Ru}(\mathrm{III})$, coordenado ao radical, py ${ }^{\bullet}$

$\left[\left(\mathrm{NH}_{3}\right)_{5} \mathrm{Ru}^{\mathrm{II}} \mathrm{py}\right]^{2+} \underset{\mathrm{MLCT}}{\stackrel{\mathrm{hv}}{\longrightarrow}}\left[\left(\mathrm{NH}_{3}\right)_{5} \mathrm{Ru}^{\mathrm{III}} \mathrm{py}^{-}\right]^{2+}$

Embora, em princípio, a população do estado MLCT possa levar à oxidação do complexo, quando $\left[\mathrm{Ru}\left(\mathrm{NH}_{3}\right)_{5} \mathrm{py}\right]^{2+}$ é irradiado com luz visível não se observa a formação de produtos provenientes de oxidação ${ }^{21}$. Por outro lado, a estrutura de MLCT para o complexo com piridina sugere que o anel aromático no estado excitado é mais susceptível a reações eletrofílicas que o estado fundamental. Em experimentos de fotólise na presença de água deuterada ${ }^{21 \mathrm{c}}$ observou-se a troca eletrofílica H/D dos prótons da piridina com rendimentos quânticos muito baixos $\left(\Phi_{\mathrm{D}} \sim 0,001\right)$, mostrando que a dependência de $\Phi_{\mathrm{py}}$ com o pH não se deve à formação de um complexo com carbono protonado. Além disso, uma vez que os complexos de Ru(III) geralmente são mais inertes ${ }^{38}$ em relação à substituição que os complexos de $\mathrm{Ru}(\mathrm{II})$, não seriam esperadas reações de aquação a partir deste estado excitado. Isto acontece com o íon complexo $\left[\mathrm{Ru}\left(\mathrm{NH}_{3}\right)_{5} \mathrm{py}\right]^{3+}$, que é inerte em relação a substituição ${ }^{38 b}$ nas condições usadas na fotólise do complexo de $\mathrm{Ru}(\mathrm{II})$.

Nos complexos de aminas de rutênio(II) $\left[\mathrm{Ru}\left(\mathrm{NH}_{3}\right)_{5} \mathrm{CH}_{3} \mathrm{CN}\right]^{2+}$ e $\left[\mathrm{Ru}\left(\mathrm{NH}_{3}\right)_{6}\right]^{2+}$ descritos anteriormente, as bandas de LF não são obscurecidas por bandas de MLCT $^{25,26,36}$. Para o complexo $\left[\mathrm{Ru}\left(\mathrm{NH}_{3}\right)_{5} \mathrm{py}\right]^{2+}$ estas bandas aparecem em comprimentos de onda próximos à banda de $\mathrm{MLCT}^{19}$. Como visto anteriormente ${ }^{25,26}$, a irradiação direta nas bandas de LF resulta na substituição dos ligantes, sendo este comportamento fotoquímico consistente com a observação geral de que a irradiação nas bandas de LF de complexos $\mathrm{d}^{6}$ de spin baixo leva à fotossubstituição dos ligantes ${ }^{10 b, 39}$. Além disso, para $\left[\mathrm{Ru}\left(\mathrm{NH}_{3}\right)_{5} \mathrm{py}\right]^{2+}$, a independência dos rendimento quânticos com o $\lambda_{\text {irr }}$ sugere um estado excitado reativo comum.

Com base nestes argumentos, Ford e colaboradores ${ }^{21,22}$ propuseram o estado excitado de LF como responsável pela fotoaquação de $\mathrm{NH}_{3}$ e py em $\left[\mathrm{Ru}\left(\mathrm{NH}_{3}\right)_{5} \text { py }\right]^{2+}$. Este estado excitado seria populado a partir do estado de MLCT excitado inicialmente ${ }^{21 \mathrm{c}, 22 \mathrm{a}}$. Posteriormente ${ }^{22 \mathrm{~b}}$, estes autores propuseram o modelo fotoquímico conhecido como "tuning dos estados excitados", fundamentado na dependência da posição da banda de MLCT do complexos $\left[\mathrm{Ru}\left(\mathrm{NH}_{3}\right)_{5} \mathrm{py}-\mathrm{X}\right]^{2+}$ com os substituintes e com o solvente. Este modelo baseia-se no fato de que, enquanto que a energia das transições de MLCT é sensível aos parâmetros acima, a energia das transições de LF geralmente é insensível ao solvente ${ }^{36}$, assim como as bandas de LF para os complexos análogos de $\mathrm{Rh}$ (III) e Co(III) são insensíveis ao substituinte ${ }^{39}$. Portanto, mudando-se o substituinte $\mathrm{X}$ em py-X é possível variar a energia do estado excitado de MLCT, sem alterar a energia do estado de LF, até se obter a inversão da ordem energética destes estados excitados. Deste modo, para complexos cujo estado excitado de menor energia fosse o de MLCT, não haveria fotorreação, enquando que quando o estado excitado de menor energia fosse o de LF, o complexo seria reativo. Isto foi confirmado experimentalmente ${ }^{22 \mathrm{~b}}$ e os rendimentos quânticos para a fotoaquação de ligantes quando estes complexos são irradiados no comprimento de onda do máximo da banda de transferência de carga, $\lambda_{\max }(\mathrm{CT})$ são mostrados na tabela 1 .

Para os complexos onde $\lambda_{\max }(\mathrm{CT})<460 \mathrm{~nm}$, os rendimentos quânticos de fotoaquação são maiores que $0,02 \mathrm{~mol} /$ einstein, enquanto que, para os complexos com $\lambda_{\max }(\mathrm{CT})>460 \mathrm{~nm}$, estes rendimentos são menores que $0,002 \mathrm{~mol} /$ einstein $^{22 \mathrm{~b}}$. Com base nestes resultados, os complexos $\left[\mathrm{Ru}\left(\mathrm{NH}_{3}\right)_{5} \mathrm{py}-\mathrm{x}\right]^{2+}$ foram classificados como "reativos" e "não reativos"22b. Os complexos "reativos" são os que apresentam rendimentos quânticos de substituição relativamente altos $(\Phi>0,02 \mathrm{~mol} /$ einstein $)$ 
independentes do $\lambda_{\text {irr }}$ e máximos de absorção a $\lambda<460 \mathrm{~nm}$ em solução aquosa. Por outro lado, os complexos "não reativos" são os que apresentam rendimentos quânticos de substituição baixos $\left(\Phi<0,002 \mathrm{~mol} /\right.$ einstein), dependentes do $\lambda_{\text {irr }}$, e máximos de absorção a $\lambda>460 \mathrm{~nm}$. Estas observações levaram os autores $^{22 b}$ a propor um estado excitado de LF para as fotossubstituições observadas.

De acordo com o modelo "tuning dos estados excitados" (Figura 4), o estado excitado de menor energia para os complexos considerados reativos é de LF (Figura 4a), enquanto que o estado excitado de menor energia para os complexos considerados não reativos é MLCT (Figura 4b) ${ }^{22 b}$. A irradiação na banda de MLCT é seguida pelo decaimento para o estado excitado de LF de menor energia, a partir do qual ocorre a aquação ou a desativação não-radiativa do complexo (Figura 4a). Para os complexos "não reativos" a irradiação na banda de MLCT é seguida por desativação para o estado de menor energia de MLCT, em competição com o decaimento para os es-

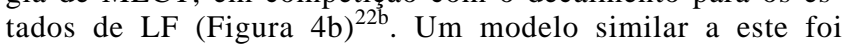
proposto para a substituição fotoquímica de py-x na série ${ }^{40}$ $\left[\mathrm{W}(\mathrm{CO})_{5} \mathrm{py}-\mathrm{x}\right]$.

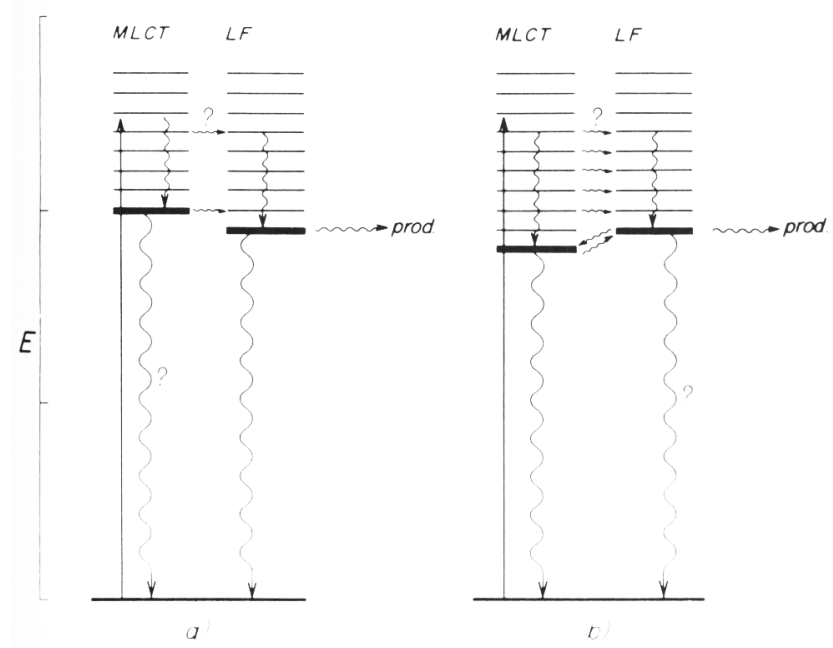

Figura 4. Diagramas de energia para explicar o comportamento fotoquímico dos complexos $\left[\mathrm{Ru}\left(\mathrm{NH}_{3}\right)_{5} \mathrm{py}-\mathrm{x}\right]^{2+}$. a) complexos "reativos"; b) complexos "não reativos". (Ref. 22b).

Em 1972, Natarajan e Endicott ${ }^{16 b}$ observaram a presença de um transiente com tempo de vida dependente do $\mathrm{pH}$ na fotólise relâmpago de $\left[\mathrm{Ru}\left(\mathrm{NH}_{3}\right)_{5} \mathrm{py}\right]^{2+}$. A velocidade de decaimento deste transiente $\left(32+6,8\left[\mathrm{H}^{+}\right]^{1} \mathrm{~s}^{-1}\right)$ foi considerada muito lenta para representar o comportamento de um estado excitado. Em adição, foi observado que, após o pulso de luz, o complexo inicial era regenerado. Com base nestes resultados, foi proposto que o mecanismo de aquação do complexo $\left[\mathrm{Ru}\left(\mathrm{NH}_{3}\right)_{5} \mathrm{py}\right]^{2+}$ dependente do $\mathrm{pH}$ deveria envolver a formação de um complexo intermediário formado pela rehibridização e protonação do nitrogênio da piridina no estado de MLCT, para dar lugar a um complexo de $\mathrm{Ru}(\mathrm{III})$ coordenado a um radical-íon de piridina $\left(\mathrm{py}^{-\bullet}\right)$. Neste intermediário o nitrogênio da piridina assume uma configuração tetraédrica passível de protonação reversível.

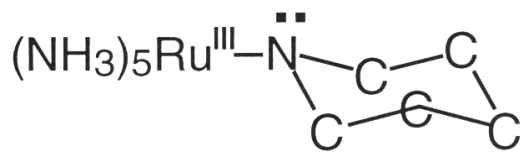

Esses autores ${ }^{16 \mathrm{~b}}$ propõem um mecanismo que não deixa claro se o caminho da reação independente do $\mathrm{pH}$ ocorre diretamente a partir do intermediário ou se há uma competição entre a transferência de elétrons de volta, ou ainda se as reações de aquação dos ligantes estão associadas a um outro estado excitado. $\mathrm{O}$ efeito da concentração de $\mathrm{H}^{+}$sobre a velocidade de decaimento do transiente é bastante coerente com a dependência de $\Phi_{\text {py }}$ com o pH da solução. No entanto, os autores propõem que o intermediário é produzido por uma distorção direta do estado de MLCT. Estas conclusões ${ }^{16 \mathrm{~b}}$ estão em total discordância com o modelo de "tuning dos estados excitados" proposto por Ford ${ }^{22 b}$.

Neste contexto, Durante e Ford ${ }^{16 \mathrm{~d}}$ realizaram experimentos de fotólise relâmpago para a série $\left[\mathrm{Ru}\left(\mathrm{NH}_{3}\right)_{5} \mathrm{py}-\mathrm{x}\right]^{2+}$, confirmando os resultados experimentais de Natarajam e Endicott ${ }^{16 \mathrm{~b}}$. Em adição, foi observada a formação de transientes de vida longa para todos os complexos que também apresentaram fotoaquação significativa dos ligantes coordenados. Uma vez que somente para os íons complexos que mostram fotoaquação significativa $\left(\Phi_{\mathrm{py}-\mathrm{x}}>0,01\right)$ foram observados transientes em quantidades apreciáveis, e sendo estes os que segundo esses autores $^{22 \mathrm{~b}}$ têm um estado de LF como o estado de menor energia $^{22 b}$, concluiu-se que esse estado de LF é o precursor imediato dos transientes. Foi então proposto ${ }^{16 \mathrm{~d}}$ que o transiente é um intermediário (não um estado excitado) com o anel da piridina ligado a $\mathrm{Ru}\left(\mathrm{NH}_{3}\right)_{5}$ de tal maneira que o par de elétrons do nitrogênio fica livre para a protonação reversível.

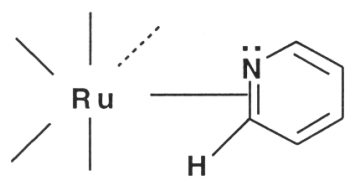

Um complexo $\pi$ deste tipo foi descrito recentemente ${ }^{20 a}$ para explicar a reação de isomerização de ligação que ocorre ao se reduzir o complexo $\left[\mathrm{Ru}^{\mathrm{III}}\left(\mathrm{NH}_{3}\right)_{5}(\mathrm{NHCOpy})\right]^{3+}$ a $\left[\mathrm{Ru}^{\mathrm{II}}\left(\mathrm{NH}_{3}\right)_{5}\right.$ $(\mathrm{NHCOpy})]^{2+}$. Após a redução, o complexo de $\mathrm{Ru}(\mathrm{II})$ inicialmente coordenado ao nitrogênio da amida sofre isomerização, formando o complexo pentaaminarutênio(II) coordenado à isonicotinamida pelo nitrogênio piridínico. Inferiu-se que esta isomerização ocorre via um intermediário em que o $\mathrm{Ru}(\mathrm{II})$ se encontra coordenado por ligações $\pi$ ao anel aromático. Intermediários similares também foram propostos para algumas reações de complexos de $\mathrm{Os}^{41}$.

Também foram realizados estudos de espectroscopia de ressonância Raman ${ }^{16 e}$ do estado excitado de dois compostos "modelos" da série $\left[\mathrm{Ru}\left(\mathrm{NH}_{3}\right)_{5} \text { py-x }\right]^{2+}:\left[\mathrm{Ru}\left(\mathrm{NH}_{3}\right)_{5}(\text { acpy })\right]^{2+}$, (acpy $=4$-acetilpiridina $)$, e $\left[\mathrm{Ru}\left(\mathrm{NH}_{3}\right)_{5} \mathrm{py}\right]^{2+}$. O espectro de ressonância Raman do estado excitado de $\left[\mathrm{Ru}\left(\mathrm{NH}_{3}\right)_{5} \text { (acpy) }\right]^{2+}\left(\lambda_{\max }\right.$ $(\mathrm{CT})=524 \mathrm{~nm}$ ) apresentou picos atribuídos ao radical acpy ${ }^{-}$, consistentes com o modelo proposto por Ford ${ }^{22 \mathrm{~b}}$ para os complexos não reativos. Para o complexo $\left[\mathrm{Ru}\left(\mathrm{NH}_{3}\right)_{5} \mathrm{py}\right]^{2+}$, quando excitado a $340 \mathrm{~nm}$ não foram observados os picos esperados do radical piridina $(\sim 340 \mathrm{~nm})$. Os autores argumentam que a presença do cátion metálico poderia deslocar esta transição para abaixo de $300 \mathrm{~nm}$ de forma a dificultar sua detecção. Este resultado não prova que o estado de LF esteja posicionado abaixo do estado de MLCT, mas é coerente com o modelo de "tuning dos estados excitados" proposto por Ford ${ }^{22 b}$. Por outro lado, Creutz e colaboradores ${ }^{16 f}$ descreveram alguns estudos de espectroscopia de absorção de transientes para uma série de complexos de $\mathrm{Ru}(\mathrm{II})$, incluindo $\left[\mathrm{Ru}\left(\mathrm{NH}_{3}\right)_{5} \mathrm{py}\right]^{2+}$, obtendo tempos de vida muito curtos ( $\tau \sim 20 \mathrm{ps}$ ) para o estado de MLCT de $\left[\mathrm{Ru}\left(\mathrm{NH}_{3}\right)_{5} \mathrm{py}\right]^{2+}$. O estado excitado de LF não foi observado diretamente, mas inferiu-se que o seu tempo de vida deveria ser curto também.

O comportamento fotoquímico dos complexos $\left[\mathrm{Ru}\left(\mathrm{NH}_{3}\right)_{5}\right.$ py- $\mathrm{x}]^{2+}$, quando interpretado de acordo com o modelo de "tuning dos estados excitados" (Figura 4), ilustra bem as diferenças existentes entre os estados inicialmente formados por irradiação e o 
estado responsável pela fotorreação. Para estes complexos, os espectros de absorção no visível são caracterizados por bandas de MLCT largas e intensas, fazendo com que as bandas de LF sejam apenas inferidas, uma vez que estão encobertas pela banda de MLCT. No entanto, as fotólises ${ }^{22 b}$ e os estudos de fotólise relâmpago ${ }^{16 \mathrm{~d}}$ para $\left[\mathrm{Ru}\left(\mathrm{NH}_{3}\right)_{5} \mathrm{py}\right]^{2+}$ indicam que os estados de LF devem ser os responsáveis pela reatividade fotoquímica destes complexos. Esses experimentos mostram também que alterações no complexo, como a substituição num sítio do ligante ou a mudança de solvente, podem modificar a ordem energética dos estados de menor energia e alterar a reatividade fotoquímica do complexo.

Como os complexos de $\mathrm{Ru}(\mathrm{II})$ e $\mathrm{Ru}(\mathrm{III})$ são inertes em relação à substituição térmica ${ }^{15,38}$, não há razão para se esperar que o estado de MLCT apresente reações de substituição de ligantes. Por outro lado, a população de um orbital antiligante na ligação metal-ligante, como na transição de campo ligante de menor energia ${ }^{1,3} \mathrm{~T}_{1 \mathrm{~g}}\left(\mathrm{t}_{2 \mathrm{~g}}{ }^{5} \mathrm{e}_{\mathrm{g}}{ }^{1}\right) \leftarrow{ }^{1} \mathrm{~A}_{1 \mathrm{~g}}\left(\mathrm{t}_{2 \mathrm{~g}}{ }^{6}\right)$ na simetria octaédrica, leva a um complexo de Ru(II) lábil em relação à substituição. Sob este ponto de vista, o modelo de "tuning dos estados excitados " proposto por Ford é coerente com as expectativas de labilização dos estados de MLCT e de LF. Este modelo tem sido muito usado qualitativamente ${ }^{16 \mathrm{~d}, \mathrm{e}, \mathrm{f}, 22,40}$, no entanto, a falta de dados espectroscópicos e fotofísicos tem dificultado uma proposta quantitativa. Frente a impossibilidade de se observar diretamente ${ }^{16 \mathrm{f}, 22}$ a luminescência do complexo $\left[\mathrm{Ru}\left(\mathrm{NH}_{3}\right)_{5} \mathrm{py}\right]^{2+}$, o que impede a determinação dos níveis de energia dos estados precursores da fotoquímica de $\left[\mathrm{Ru}\left(\mathrm{NH}_{3}\right)_{5}\right.$ py $]^{2+}$, foram efetuados recentemente ${ }^{27}$ experimentos de sensibilização e supressão deste complexo para avaliar quantitativamente o modelo proposto por Ford ${ }^{22 b}$.

Neste estudo ${ }^{27}$, uma série de compostos orgânicos foram usados como sensibilizadores para as reações de aquação do íon complexo $\left[\mathrm{Ru}\left(\mathrm{NH}_{3}\right)_{5} \mathrm{py}\right]^{2+}$. Os sensibilizadores foram escolhidos de acordo com as energias estimadas dos estados excitados de $\left[\mathrm{Ru}\left(\mathrm{NH}_{3}\right)_{5} \mathrm{py}\right]^{2+}$. O espectro de reflectância difusa de $\left[\mathrm{Ru}\left(\mathrm{NH}_{3}\right)_{5} \mathrm{py}\right]^{2+}$ no estado sólido apresenta um pico a $\sim 410 \mathrm{~nm}$ e um ombro largo em $\sim 385 \mathrm{~nm}$, que é o mesmo comprimento de onda da banda ${ }^{26,36}$ de $\mathrm{LF}$ de menor energia do $\left[\mathrm{Ru}\left(\mathrm{NH}_{3}\right)_{6}\right]^{2+}$ $\left(\lambda=390 \mathrm{~nm}, \varepsilon=35 \mathrm{M}^{-1} \mathrm{~cm}^{-1}\right)$, atribuída à transição singlete ${ }^{1} \mathrm{~T}_{1 \mathrm{~g}} \leftarrow{ }^{1} \mathrm{~A}_{1 \mathrm{~g}}$. Fazendo-se a mesma atribuição para $\left[\mathrm{Ru}\left(\mathrm{NH}_{3}\right)_{5}\right.$ py $]^{2+}$, pode-se concluir ${ }^{27}$ que o complexo $\left[\mathrm{Ru}\left(\mathrm{NH}_{3}\right)_{5} \mathrm{py}\right]^{2+}$ deve apresentar uma transição de LF na mesma região espectroscópica do íon $\left[\mathrm{Ru}\left(\mathrm{NH}_{3}\right)_{6}\right]^{2+}$, mas obscurecida pela banda mais intensa de MLCT. Para a avaliação das energias dos estados excitados reativos, $\mathrm{E}_{\mathrm{o}-\mathrm{o}}$, foi levado em consideração ${ }^{27}$ que o máximo de absorção observado no visível (i.e., a transição "vertical") não corresponde à diferença de energia entre o estado excitado e o estado fundamental, mas inclui uma quantidade razoável de energia vibracional, $\lambda^{*}$, relacionada a $\mathrm{E}_{\mathrm{o}-\mathrm{o}}$ por $\mathrm{E}_{\mathrm{op}}=\mathrm{E}_{\mathrm{o}-\mathrm{o}}-\lambda^{*}$, onde $\mathrm{E}_{\mathrm{op}}$ é a energia "vertical". Assim, os valores de $E_{0-o}$ para os estados excitados singlete e triplete de LF foram estimados usando-se o parâmetro $\Delta_{\mathrm{o}}=26800 \mathrm{~cm}^{-1} \mathrm{da}$ teoria de campo ligante ${ }^{42}$ e a relação $\lambda^{*}=4,5 \times 10^{-6}\left(\Delta_{\mathrm{o}}\right)^{2}$. Desta forma, as energias dos estados excitados singlete e triplete de menor energia de campo ligante ${ }^{1} \mathrm{LF}$ e ${ }^{3} \mathrm{LF}$ para o complexo $\left[\mathrm{Ru}\left(\mathrm{NH}_{3}\right)_{5} \mathrm{py}\right]^{2+}$ foram estimadas em $21022 \mathrm{~cm}^{-1} \mathrm{e}$ $17677 \mathrm{~cm}^{-1}$, respectivamente. Da energia do estado de MLCT de Franck-Condon obtida diretamente pela absorção da luz $\left(24500 \mathrm{~cm}^{-1}\right)$, e usando para $\lambda^{*}$ a diferença de energia entre os estados singlete e triplete correspondente ao $\left[\mathrm{Ru}(\mathrm{bipy})_{3}\right]^{2+}$ $\left(3600 \mathrm{~cm}^{-1}\right)^{43}$, foram calculados os valores de $E_{0-o}$ para os estados excitados singlete e triplete de MLCT, como sendo 21700 $\mathrm{cm}^{-1}$ e $19000 \mathrm{~cm}^{-1}$, respectivamente. A figura 5 mostra o diagrama de energia proposto para os estados excitados do complexo e dos compostos orgânicos usados.

Foi observado ${ }^{27}$ que a irradiação de soluções aquosas de $\left[\mathrm{Ru}\left(\mathrm{NH}_{3}\right)_{5} \mathrm{py}\right]^{2+}$ na presença dos corantes rodamina-B $\left(\lambda_{\max }=\right.$ $554 \mathrm{~nm})$ ou safranina-T $\left(\lambda_{\max }=520 \mathrm{~nm}\right)$ com luz de $520 \mathrm{~nm}$

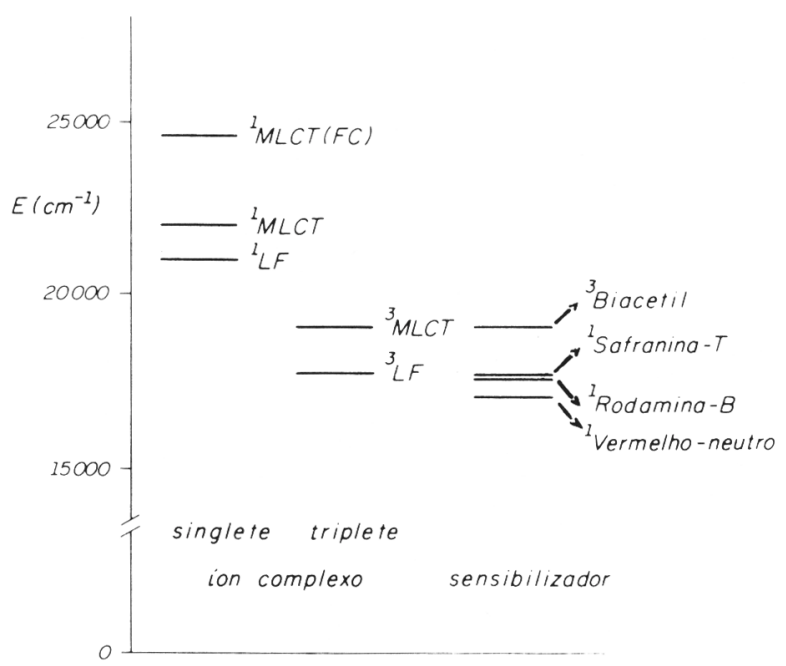

Figura 5. Diagrama de níveis de energia para os estados excitados do complexo $\left[\mathrm{Ru}\left(\mathrm{NH}_{3}\right)_{5} \text { py }\right]^{2+}$ e para os sensibilizadores orgânicos.

${ }^{1} \mathrm{MLCT}(\mathrm{FC})=$ estado de Franck-Condon de MLCT; ${ }^{1} \mathrm{MLCT}=$ estado excitado singlete de menor energia de $M L C T ;{ }^{3} \mathrm{MLCT}$ = estado excitado triplete de menor energia de $M L C T ;{ }^{1} \mathrm{LF}=$ estado excitado triplete de menor energia de $L F ;{ }^{3} \mathrm{LF}=$ estado excitado triplete de menor energia de LF. ${ }^{1}$ Vermelho-neutro, ${ }^{1}$ Rodamina-B, ${ }^{1}$ Safranina-T = estados excitados singlete de menor energia para estes corantes; ${ }^{3}$ biacetil $=$ estado triplete do biacetilo.

resultou na aquação do complexo $\left(\Phi_{\mathrm{NH} 3}=0,095\right.$ e $\Phi_{\mathrm{py}}=0,078$ mol/einstein, para o corante rodamina-B). A banda de absorção do corante não foi alterada durante as fotólises, indicando que estes corantes são estáveis fotoquimicamente na presença do complexo. A observação de que os mesmos produtos e a mesma razão de rendimentos quânticos foram observados em experimentos de sensibilização e de fotólise direta, indica que todos os produtos devem originar-se de um mesmo estado excitado. Através de experimentos de fotólise relâmpago, Ford e colaboradores ${ }^{16 \mathrm{~d}}$ determinaram o rendimento quântico aparente, $\Phi_{\text {ap }}$, para a fotoaquação de $\left[\mathrm{Ru}\left(\mathrm{NH}_{3}\right)_{5} \text { py }\right]^{2+}$ como sendo $0,16 \mathrm{~mol} /$ einstein. A soma dos rendimentos quânticos obtidos por sensibilização ${ }^{27}\left(\Phi^{\mathrm{S}}{ }_{\mathrm{NH} 3}+\Phi_{\text {py }}^{\mathrm{S}}\right)=0,18$ foi quase o dobro do obtido por fotólise direta ${ }^{21,22}, \Phi_{\mathrm{NH} 3}+\Phi_{\mathrm{py}}=0,10$. Mas 0,18 mol/einstein é similar ao valor de rendimento quântico aparente obtido por fotólise relâmpago ${ }^{16 d}$. Portanto, pode-se concluir ${ }^{27}$ que os mesmos estados excitados são alcançados por fotólise direta e sensibilizada e que todos os produtos devem originarse desses estados. Considerando-se que os estados excitados singlete dos corantes rodamina-B $\left(17452 \mathrm{~cm}^{-1}\right)$ e safranina-T $\left(17690 \mathrm{~cm}^{-1}\right)$, assim como a fosforescência do biacetilo (19000 $\mathrm{cm}^{-1}$ ), foram suprimidos pelo complexo, e que não foi observada a supressão da fluorescência do corante vermelho neutro $\left(16900 \mathrm{~cm}^{-1}\right)$, pode-se concluir que a energia do estado spinórbita sensibilizado que leva às reações de fotoaquação esteja entre $16900 \mathrm{~cm}^{-1}$ e $17700 \mathrm{~cm}^{-1}$. Estes resultados são consistentes com o modelo de "tuning dos estados excitados" proposto por Ford ${ }^{22 b}$.

\section{SUMÁRIO}

As seções anteriores fazem uma revisão dos estudos experimentais e teóricos descritos na literatura durante as duas últimas décadas, no que concerne às reações fotoquímicas dos complexos pentaaminas de rutênio(II) com ligantes $\pi$ insaturados. Os comportamentos fotoquímicos observados podem ser descritos como sendo o produto de uma reação fotoquímica primária ou o resultado da formação de intermediários provenientes de reações fotorredox ou de substituição. Como vimos, o conhecimento dos mecanismos destas reações foi se tornando 
cada vez mais sofisticado. Novas técnicas, especialmente laser de pulso, deram a possibilidade de se determinar as propriedades dos estados excitados e medir as velocidades das reações fotoquímicas. Certamente, os avanços instrumentais que temos observado, assim como a aplicação das técnicas fotoquímicas rotineiras, prometem uma evolução grande na obtenção das informações necessárias para a interpretação dos mecanismos e propriedades dos estados excitados dos complexos de metais de transição.

\section{REFERÊNCIAS}

1. Balzani, V.; Carassiti, V.; Photochemistry of Coordination Compounds; Academic Press, London, 1970

2. Adamson, A. W.; Waltz, W. L.; Zinatto, E.; Watts, D. W.; Fleischauer, P.; Lindholm, R. D.; Chem. Rev. 1968, $68,541$.

3. Adamson, A. W.; J. Phys. Chem. 1967, 71, 798.

4. Vogler, A.; Adamson, A. W.; J. Am. Chem. Soc. 1968, 90, 5943

5. Kane Maguire, N. A. P.; Langford, C. H.; Chem. Commun. 1971, 895.

6. (a) Sastri, V. S.; Langford, C. H.; J. Am. Chem. Soc. 1969, 91, 7533. (b) Porter, G. B.; J. Am. Chem. Soc. 1969, 91, 3980. (c) Scandola, M. A.; Scandola, F.; J. Am. Chem. Soc. 1970, 92, 7278. (d) Chen, S.; Porter, G. B.; Chem. Phys. Lett. 1970, 8, 41. (e) Balzani, V.; Ballardini, R.; Gandolfi, M. T.; Moggi, L.; J. Am. Chem. Soc. 1971, 93, 339. (f) Scandola, M. A.; Scandola, F.; J. Am. Chem. Soc. 1972, 94, 1805.

7. Balzani, V.; Moggi, L.; Manfrin, M. F.; Bolletta, F.; Coord. Chem. Rev. 1975, 15, 321.

8. Balzani, V.; Bolletta, F.; Gandolfi, M. T.; Maestri, M.; Top. Curr. Chem. 1978, 75, 1

9. (a) Wrighton, M. S.; Acc. Chem. Res. 1979, 12, 303. (b) Balzani, V.; Scandola, F. em Photochemical Conversion and Storage of Solar Energy; (J. S. Connolly, ed.); Academic Press; New York, 1981. (c) Scandola, F.; Balzani, V.; J. Chem. Ed. 1983, 60, 814. (d) Kavarnos, G. J.; Turro, N.J. Chem. Rev. 1986, 86, 401. (e) Scandola, F.; Indelli, M. T.; Chiorboli, C.; Bignozzi, C. A.; Top. Curr. Chem. 1990, 158, 73. (f) Balzani, V.; Juris, A.; Venturi, M.; Chem. Rev. 1996, 96, 759.

10. (a) Ford, P. C.; Petersen, J. D.; Hintze, R. E.; Coord. Chem. Rev. 1974, 14, 67. (b) Ford, P. C.; Rev. Chem. Int. 1979, 2, 267.

11. (a) Watts, R. J.; J. Chem. Ed. 1983, 60, 834. (b) Juris, A.; Balzani, V.; Barigelletti, F.; Campagna, S.; Belser, P.; Vonzeewsky, A.; Coord. Chem. Rev. 1988, 84, 85. (c) Kalyanasundaram, K.; Photochemistry of Polypyridine and Porphyrin Complexes; Academic Press; San Diego, CA, 1992.

12. Geoffroy, G. L.; Wrighton, M. S.; Organometallic Photochemistry; Academic Press; New York, 1979.

13. (a) Ferraudi, G. J.; Elements of Inorganic Photochemistry, Wiley Interscience; New York, 1988. (b) Vogler, A.; Yersin, H. (eds); Photophysics and Photochemistry of Coordination Compounds, Springler-Verlag, Berlin, 1988. (c) Balzani, V.; Moggi, L.; Coord. Chem. Rev. 1990, 97, 313. (d) Balzani, V.; Scandola, F.; Supramolecular Photochemistry; Horwood; Chichester, U.K., 1991.

14. (a) Wayne, R. P.; Principles and Applications of Photochemistry; Oxford Science Publ.; New York, 1988. (b) Joachin, C.; Launay, J. P.; Chem. Phys. 1986, 109, 93. (c) Veno, A.; Takahashi, K.; Osa, T.; J. Chem. Soc. Chem. Commun. 1981, 94. (d) Monti, S.; Flamigni, L.; Martelli, A.; Bortolus, P.; J. Phys. Chem. 1988, 92, 4447. (e) Sykora, J.; Sima, J.; Coord. Chem. Rev. 1990, 107, 1. (f) Karas, J. L.; Lieber, C. M.; Gray, H. B.; J. Am. Chem.
Soc. 1988, 10, 599. (g) Bjerrum, M. J.; Casimiro, D. R.; Chang., I. J.; Di Bilio, A. J.; Gray, H. B.; Hill, M. G.; Langen, R.; Mines, G. A.; Skov, L. K.; Winkler, J. R.; Wuttke, D. S., J. Bioenerg. Biomembr., 1995, 27, 295.

15. (a) Katakis, D.; Gordon, G. "Mechanisms of Inorganic Reactions", Wiley, New York, 1987. (b) Wilkinson, R. G.; Kinetics and Mechanisms of Reactions of Transition Metal Complexes; 2nd Ed.; VCH; Weinheim, 1991.

16. (a) Adamson, A. W. J. Chem. Ed. 1983, 60, 797. (b) Natarajan, P.; Endicott, J. F.; J. Am. Chem. Soc. 1972, 94, 5909. (c) Toma, H. E.; Santos, P. S.; Inorg. Chim. Acta, 1977, 24, L61. (d) Durante, V. A.; Ford, P. C.; Inorg. Chem. 1979, 18, 588. (e) Chung, Y. C.; Leventes, N.; Wagner, P. J.; Leroi, G. E.; J. Am. Chem. Soc. 1985, 107, 1414. (f) Winkler, J. R.; Nietzel, T. L.; Creutz, C.; Sutin, N.; J. Am. Chem. Soc. 1987, 109, 2381. (g) Johnson, S.R.; Westmoreland, T. D.; Gaspar, J. V.; Barqawi, K. R.; Meyer, T. J.; Inorg. Chem. 1988, 27, 3195. (h) Crawford, B. A.; Ondrias, M. R.; J. Phys. Chem. 1989, 93, 5055.

17. (a) Adamson, A. W.; Fleischauer, P.; Eds., Concepts of Inorganic Photochemistry; Wiley; New York, 1975. (b) Wrighton, M. S.; Top. Cur. Chem. 1976, 37, 65. (c) Monsted, L.; Monsted, O.; Coord. Chem. Rev. 1989, 94, 109. (d) Skibsted, L. H.; Coord. Chem. Rev. 1989, 94, 151.

18. (a) Ford, P. C.; Coord. Chem. Rev. 1982, 44, 61. (b) Ford, P. C.; Wink, D.; DiBenedetto, T.; Prog. Inorg. Chem. 1983, 30, 213.

19. (a) Ford, P. C. ; Rudd, D.; Gaunder, R.; Taube, H.; J. Am. Chem. Soc. 1968, 90, 1187. (b) Creutz, C.; Taube, H.; J. Am. Chem. Soc. 1973, 95, 1086. (c) Shepherd, R. E.; Taube, H.; Inorg. Chem. 1973, 12, 1392. (d) Curtis, J. C.; Sullivan, B. P.; Meyer, T.; Inorg. Chem. 1983, 22, 224. (e) Johnson, C. R.; Shepherd, R. E.; Inorg. Chem. 1983, 22, 2439. (f) Lavalle, D. K.; Fleisher, E. B.; J. Am. Chem. Soc. 1972, 94, 2583. (g) Stavrew, K. K.; Zerner, M. C.; Meyer, T. J.; J. Am. Chem. Soc. 1995, 117, 8684.

20. (a) Chou, M. E.; Brunschwig, B. S.; Creutz, C.; Sutin, N.; Inorg. Chem. 1992, 31, 5347. (b) Rocha, Z. N.; Chiericato, G.; Tfouni, E.; Inorg. Chem. 1994, 33, 4619. (c)Tomita, A.; Sano, M.; Inorg. Chem. 1994, 33, 5825. (d) Chou, M. H.; Szalda, D. J.; Creutz, C.; Sutin, N.; Inorg. Chem. 1994, 33, 1674.

21. (a) Ford, P. C.; Stuerner, D. H.; McDonald, D. P.; J. Am. Chem. Soc. 1969, 91, 6209. (b) Ford, P. C.; Chaison, D. A.; Stuerner, D. H.; Chem. Commun. 1971, 530. (c) Chaison, D. A.; Hintze, R. E.; Stuerner, D. H.; Petersen, P. D.; McDonald, D. P.; Ford, P. C.; J. Am. Chem. Soc. 1972, 94, 6665 .

22. (a) Malouf, G.; Ford, P. C.; J. Am. Chem. Soc. 1974, 96, 601. (b) Malouf, G.; Ford, P. C.; J. Am. Chem. Soc. 1977, 99, 7213.

23. (a) Tfouni, E.; Ford, P. C.; Inorg. Chem. 1980, 19, 72. (b) Pavanin, L. A.; Rocha, Z. N.; Giesbrecht, E.; Tfouni, E.; Inorg. Chem. 1991, 30, 2185.

24. Hintze, R. E.; Ford, P .C.; Inorg. Chem. 1975, 14, 1211

25. Hintze, R. E.; Ford, P. C.; J. Am. Chem. Soc. 1975, 97, 2664

26. Matsubara, T.; Ford, P. C.; Inorg. Chem. 1978, 17, 1747

27. (a) Carlos, R. M.; Tese de doutorado; Instituto de Química, UNESP; Araraquara, 1995. (b) Carlos, R. M.; Neumann, M. G.; Tfouni, E.; Inorg. Chem., 1996, 35, 2229.

28. Carlos, R. M.; Neumann, M. G.; Tfouni, E.; Photochem. Photobiol., no prelo, 1996.

29. (a) Creutz, C.; Kroger, P.; Matsubara, T.; Netzel, T.L.; Sutin, N. J.; J. Am. Chem. Soc. 1979, 101, 5442. (b) Schanze, K. S.; Meyer, T. J.; Inorg. Chem. 1985, 24, 
2121. (c) Shi-A; Anson, F. C. Inorg. Chim. Acta, 1994, $225,215$.

30. Clarke, R. E.; Ford, P. C.; Inorg. Chem. 1970, 9, 227.

31. Treitel, I. M.; Food, M. T.; Marsh, R. E.; Gray, H. B.; J. Am. Chem. Soc. 1969, 91, 6512.

32. Durante, V. A.; Ford, P. C.; J. Am. Chem. Soc. 1975, 97, 6898.

33. Pereira, M. S.; Malin, J. M.; Inorg. Chem. 1974, 13, 386.

34. Sigwart, C.; Spence, J.; J. Am. Chem. Soc. 1969, 91, 3391.

35. Baxendale, J. H.; Mulazzani, Q. G.; J. Inorg. Nucl. Chem. 1971, 33, 823.

36. Matsubara, T. C.; Eufrima, S.; Metiu, H. I.; Ford, P. C.; J. Chem. Soc. Faraday Trans. 2; 1979, 75, 390.
37. Allen, R. J.; Ford, P. C.; Inorg. Chem. 1972, 11, 679.

38. (a) Matsubara, T.; Ford, P. C.; Inorg. Chem. 1976, 15, 1107. (b) Sexton, D. A.; Curtis, J. C.; Cohen, H.; Ford, P. C.; Inorg. Chem. 1984, 23, 49. (c) Rudd, D. P.; Taube, H.; Inorg. Chem. 1978, 17, 1747.

39. (a) Petersen, J. D.; Watts, R. J.; Ford, P. C.; J. Am. Chem. Soc. 1976, 98, 3188. (b) Nordmeyer, F.; Taube, H.; J. Am. Chem. Soc.; 1968, 90, 1162.

40. Moralejo, C.; Langford, C. H.; Inorg. Chem. 1991, 30, 567. 41. Taube, H.; Pure Appl. Chem. 1991, 63, 651.

42. Lever, A. B. P.; Inorganic Electronic Spectroscopy; Elsevier; New York, 1985.

43. Kober, E. M.; Meyer, T.J .; Inorg. Chem. 1982, 21, 3697. 